\title{
Magnetic excitations in soft X-ray RIXS: recent developments
}

\author{
S. Fatale, S. Moser and M. Grioni \\ Institute of Condensed Matter Physics, Ecole Polytechnique Fédérale Lausanne (EPFL), CH- \\ 1015 Lausanne, Switzerland
}

\begin{abstract}
Resonant inelastic X-ray scattering (RIXS) has rapidly become a mature spectroscopic technique. In particular, taking advantage of an improved energy resolution, a series of experiments performed over the past 5 years have established soft X-ray RIXS as a sensitive, quantitative tool to investigate magnetic excitations in solids, complementary to more traditional probes such as neutron scattering. Most of the work has targeted cuprate systems, and the focus has progressively shifted from the antiferromagnetic insulating parent compounds to the superconducting part of the phase diagram. Here we review the most recent results, and the outstanding questions they raise.
\end{abstract}


Resonant inelastic X-ray scattering (RIXS) is a photon-in photon-out synchrotron based spectroscopy based on a coherent second-order optical process. It can be defined as the X-ray analogue of conventional Raman or, equivalently, an X-ray energy-loss experiment [1-4]. The "resonant" attribute refers to the fact that the experiment is performed at a characteristic absorption edge of the material, which confers it chemical, site, and orbital selectivity. The theoretical foundations of RIXS were laid already at the dawn of quantum mechanics, by Kramers and Heisenberg who first derived the double differential cross section. However, experimental progress had to wait for the advent of intense and tunable X-ray beams at synchrotron sources, and for technical advances in X-ray optics and detectors, especially in the challenging soft X-ray range. The state of the art of soft X-ray RIXS at the turn of the century is well captured by a JES special issue [5]. RIXS was shown to probe charge-neutral excitations as opposed to charged excitations probed by photoemission - at the eV scale. RIXS with hard Xrays is also rapidly developing, and complementary to the soft version [6]. Nevertheless, the technical aspects, and to some extent also the focus, are somewhat different, namely concerning the possibility of studying magnetic excitations. Therefore only soft X-ray RIXS will be covered here.

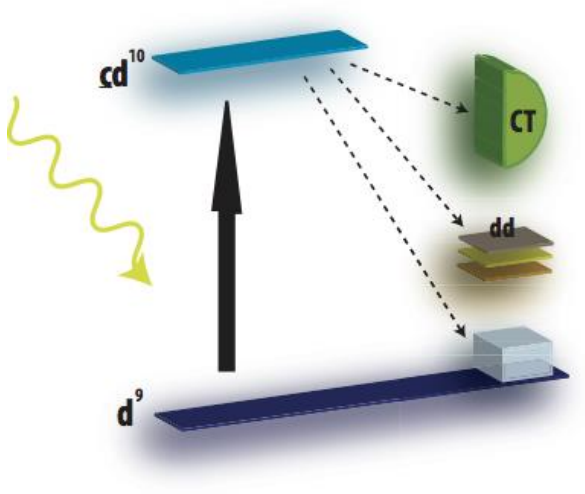

Figure 1. Schematic view of the RIXS process at the $\mathrm{L}_{3}(2 \mathrm{p} \rightarrow 3 \mathrm{~d})$ edge of a $\mathrm{Cu}^{2+}$ ion. Charge-transfer (CT), crystal-field (dd) and collective (grey box) excited states are shown.

The RIXS process is schematically illustrated in Fig. 1 for the important case of the $\mathrm{Cu}_{3}$ edge in a cuprate material. The incident photon is resonantly absorbed by the $\mathrm{Cu}^{2+}$ ion in a (mostly) $\mathrm{d}^{9}$ configuration and a $2 \mathrm{p}_{3 / 2}$ core electron is promoted to the empty $3 \mathrm{~d}$ orbital. The intermediate state $2 \mathrm{p}^{5} 3 \mathrm{~d}^{10}$ can then decay radiatively back to the ground state, or to one of the possible excited, charge-neutral, final states. The excitation energy corresponds to an energy loss for the scattered photon. The final states probed by RIXS are of various nature. They include electronhole excitations within the $d^{9}$ configurations, i.e. states where the single $3 d$ hole has been scattered from the initial $\left(\mathrm{x}^{2}-\mathrm{y}^{2}\right)$ to a different crystal-field (CEF) orbital (dd in the figure), or charge-transfer (CT) excitations where the hole moves to an orbital of the ligand, with a final state $\mathrm{d}^{10} \underline{\mathrm{L}}$ configuration. These electronic excitations occur in the $1-10 \mathrm{eV}$ energy range, and were readily identified in early RIXS work [5]. RIXS, on the other hand, is also sensitive to collective - phonon or magnon - excitations [3], which occur at energies well below $1 \mathrm{eV}$, in the mid-infrared range (MIR). Such low-energy loss features could only be identified after the commissioning of the SAXES soft X-ray RIXS instrument at the ADRESS beamline of the Swiss Light Source [7] enabled a large improvement of the experimental resolution. While in 
most cases phonons still remain out of reach, the observation and characterization of spin wave excitations already had a considerable impact in the field of non-conventional superconductivity and quantum magnetism, and rapidly changed the focus of RIXS research over the past 5 years. Here we will specifically address the rapidly expanding literature of experiments that specifically target the magnetic response.

\section{- Theoretical predictions and early experimental observations}

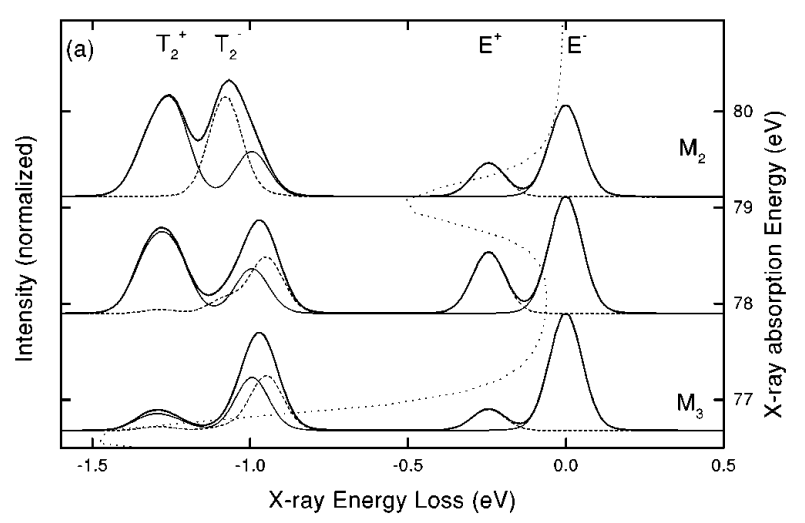

Figure 2. Calculated $\mathrm{Cu} \mathrm{M} \mathrm{M}_{2,3}$ RIXS spectra for a $\mathrm{Cu}^{2+}$ ion in octahedral symmetry, in a magnetically ordered material. The incident light is linearly polarized. Solid and dashed lines refer to two different outgoing polarizations [8].

A seminal paper by de Groot et al. [8] contains the first theoretical prediction that RIXS could be used to study magnetic excitations in solids, even if photons do not directly interact with the spin of the electron. Single spin-flip $(\Delta S=1)$ processes are normally dipole forbidden, but become allowed in a second-order process such as RIXS. At absorption edges involving a core state with nonzero angular momentum, e.g. the $\mathrm{L}_{2,3}(2 \mathrm{p})$ and $\mathrm{M}_{2,3}(3 \mathrm{p})$ edges, the total angular momentum $J$, rather than the spin, is a good quantum number, owing to the large spin-orbit coupling in the intermediate core-hole state $(19.8 \mathrm{eV}$ for $\mathrm{Cu} 2 \mathrm{p})$. Therefore, the intermediate state can decay to a final state - with no core hole - where the spin has been flipped. Total angular momentum is conserved by a change of the photon polarization. This process is more general than spin-flip Raman in the optical range, because the spin flip is enabled by the core hole, and it is possible even when the spin-orbit interaction in the valence band is quite small, as in the case of cuprate materials. By contrast, for s core states (K-edges; $1=0$ ), where spin-orbit is zero, the $\Delta S=0$ dipole selection rule holds, and only transitions involving two (or an even number of) spin flips are allowed. Figure 2 shows the calculated $\mathrm{Cu} \mathrm{M}_{2,3}(3 \mathrm{p} \rightarrow 3 \mathrm{~d})$ RIXS spectra for a divalent $\mathrm{Cu}$ ion in octahedral symmetry, in an antiferromagnetic (AFM) material. The incident photons are linearly polarized ( $\sigma$ polarization), and spectra for two different linear polarizations of the outgoing photons were separately calculated. Near the elastic peak at zero energy loss, which corresponds to transitions back to the ground state, the spectra exhibit in the $\sigma$ to $\pi$ channel a satellite at an energy loss equal to the energy required to flip the $\mathrm{Cu}$ spin in the AFM background. Even if typical experiments do not (yet) perform a polarization analysis of the scattered beam, the observation of a feature at energy loss well below the $t_{2 g} \rightarrow e_{g}$ CEF excitations, and above 
characteristic phonon energies, would establish the magnetic nature of the excitation. This remarkable prediction was not fully appreciated at the time, but proved to be an important guideline for experiments a few years later. De Groot et al. also predicted that the RIXS line shape would reproduce the magnon density of states, and that magnetic scattering would be allowed in octahedral coordination, but not in the lower-symmetry tetragonal coordination, typical of cuprates. These predictions had later to be modified in the light of new theoretical and experimental evidence.

It is interesting to notice that the single-ion, atomic calculation of Ref. [8] yields qualitatively similar results at the $\mathrm{M}_{2,3}(3 \mathrm{p})$ and $\mathrm{L}_{2,3}(2 \mathrm{p})$ edges. This could be taken as a suggestion that investigations of $3 \mathrm{~d}$ transition metal compounds should privilege technically less challenging experiments at the $\mathrm{M}$ edge, in the UV range, where higher resolving powers can be readily achieved. In reality the opposite is true, as a result of the different character of the $\mathrm{M}$ and $\mathrm{L}$ resonances, illustrated in Fig. 3 by the absorption spectra of Ni metal. The transition has a much stronger excitonic character at the L-edge, reflected in the strong and sharp edge features ("white lines"). This yields a much stronger enhancement of the L-edge RIXS signal with respect to the M-edge. Therefore, at resonance, the Raman features are comparable or even stronger than the elastic line at the L-edges. By contrast, at the $\mathrm{M}$ edges, the line shape is largely dominated by the elastic line, which includes strong non-resonant contributions [9]. Disentangling the inelastic features from the tail of the dominant elastic peak is harder despite the better experimental resolution. Moreover, especially for the early $3 \mathrm{~d}$ elements, the smaller energy separation between the $\mathrm{M}_{2}$ and $\mathrm{M}_{3}$ spin-orbit partners complicates the theoretical analysis. Furthermore, at the lower energy of the $\mathrm{M}_{2,3}$ edges, the momentum carried by the photon is extremely small, so that the available window for q-dependent studies of collective excitations is but a negligible fraction of the Brillouin zone. For these reasons progress in the study of magnetic excitations in $3 \mathrm{~d}$ transition metal compounds by soft X-ray RIXS has so far been limited, with one notable exception [10], to the L edges.

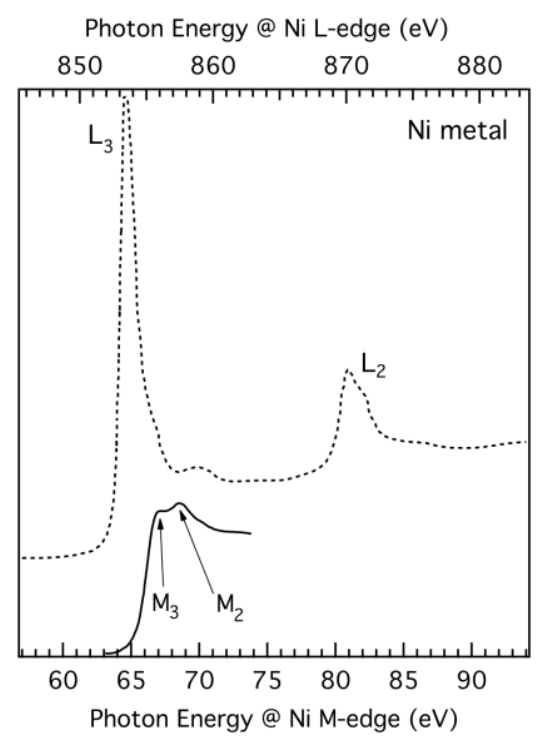

Figure 3. $\mathrm{M}_{2,3}$ and $\mathrm{L}_{2,3}$ absorption edges for $\mathrm{Ni}$ metal, arbitrarily normalized to the same edge-jump. 


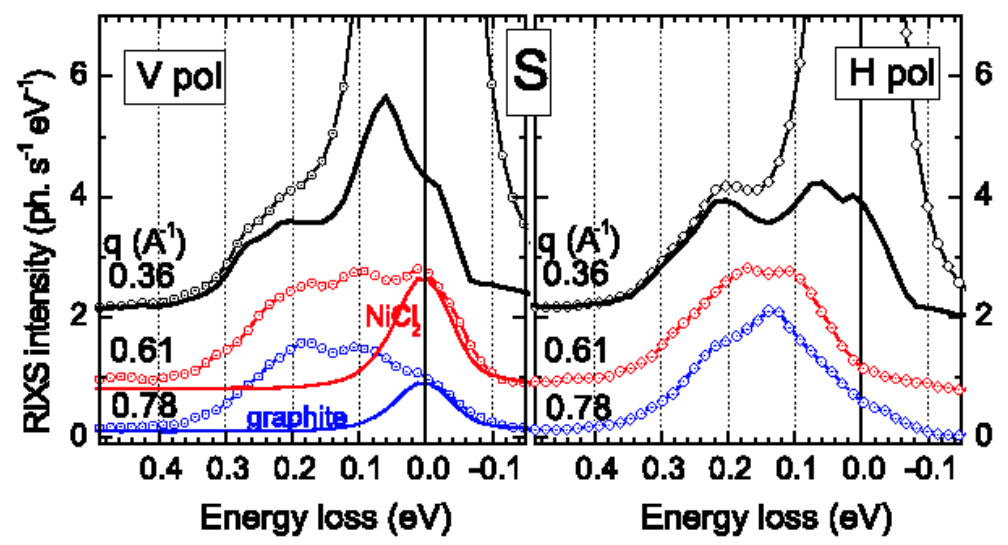

Figure 4. $\mathrm{Ni}_{3}$ edge RIXS of $\mathrm{NiO}$ for various exchanged momenta, and two polarizations of the incident beam. A resolution-limited elastic peak has been subtracted from the $\mathrm{q}=0.36 \mathrm{1} / \mathrm{A}$ spectra. The resolution-limited spectrum of $\mathrm{NiCl}_{2}$ (red line) is shown for comparison [11].

High-resolution Ni L-edge spectra of $\mathrm{NiO}$, a paradigmatic $\mathrm{AFM}\left(\mathrm{T}_{\mathrm{N}}=550 \mathrm{~K}\right)$ insulator provided the first confirmation of spin-flip excitations in RIXS [11]. Figure 4 shows $\mathrm{Ni}_{3}$ edge data measured at three different scattering angles and for two incident polarizations. All spectra exhibit loss features over an energy range of $\sim 0.3 \mathrm{eV}, \sim 4$ times larger than the energy of optical phonons in NiO. The magnetic origin of these features is confirmed by their absence in the isostructural compound $\mathrm{NiCl}_{2}$, also an AFM insulator, but with a Néel temperature, and with a corresponding characteristic magnetic energy scale, one order of magnitude smaller than in NiO. The main features at $95 \mathrm{meV}$ and $190 \mathrm{meV}$ correspond to transitions from the $\mathrm{S}=1, \mathrm{~S}_{\mathrm{Z}}=-1$ ground state of the $\mathrm{Ni}^{2+}$ ion to $\mathrm{S}_{\mathrm{Z}}=0,1$ excited states, consistent with the predictions of Ref. [8]. These data established RIXS as a probe of spin-flip excitations. However, because of the very limited sampling of momentum space, and of the presence of 12 equivalent magnetic domains, it was not possible to conclude whether these excitations were local spin-flips or rather itinerant spin waves. As discussed in the next section, these open questions would soon be settled in a different and important class of materials, the insulating cuprates.

\section{- Spin waves in two-dimensional AFM insulating cuprates}

The insulating parent compounds of the high-Tc cuprate superconductors (SC) are very interesting not only in relation with their doped counterparts. They can often be considered as practical realizations of spin- $1 / 2$ square-lattice Heisenberg AFMs, a class of systems of strong interest in the field of quantum magnetism. Moreover, the quasi-two dimensional (2D) structure and magnetic structure, and the large exchange energy $J \sim 0.13 \mathrm{eV}$, are well suited for investigations of the spin waves dispersion by RIXS. Indeed, the 2D momentum space can be sampled at fixed scattering angle and transferred momentum $\mathbf{Q}$ - the geometry of most RIXS experiments - simply by rotating the sample, thus changing the in-plane projection $\mathbf{Q}_{/ /}$of $\mathbf{Q}$. The typical experimental conditions at the $\mathrm{Cu} \mathrm{L}_{3}$ edge allow almost the complete magnetic Brillouin zone (BZ) to be covered. 
The first such Q-space survey of the antiferromagnetic cuprates $\mathrm{La}_{2} \mathrm{CuO}_{4}$ (LCO) and $\mathrm{CaCuO}_{2}$ (CCO) yielded clear inelastic features dispersing upwards from $(0,0)$ to $\sim 350 \mathrm{meV}$ near the $(\pi, 0)$ zone boundary [12], and conclusively demonstrated the collective nature of these excitations. Clear dispersive features in the RIXS spectra show that the local spin flip created in the scattering process is projected onto the eigenstates - the spin waves - of the periodic AFM solid or, equivalently, that the interaction with the incident beam creates coherent excitations at all sites. The dispersing features were at first interpreted as belonging to the two-magnon $(\Delta S=0)$ continuum, but it was soon realized that the largest contribution to the cross section is actually due to single magnons. Indeed the calculated dispersion, as well as the momentum and polarization dependence of the magnon intensity, reproduces well the experimental results [1315]. Therefore, RIXS can map pure coherent spin excitations, and reveal not just the magnon density of states [8], but the full dispersion. Moreover, theory confirmed that in tetragonal symmetry single magnon excitations are only forbidden if the spin is directed along the c-axis [13], but allowed for other orientations, namely for the in-plane (110) orientation, typical of cuprates.
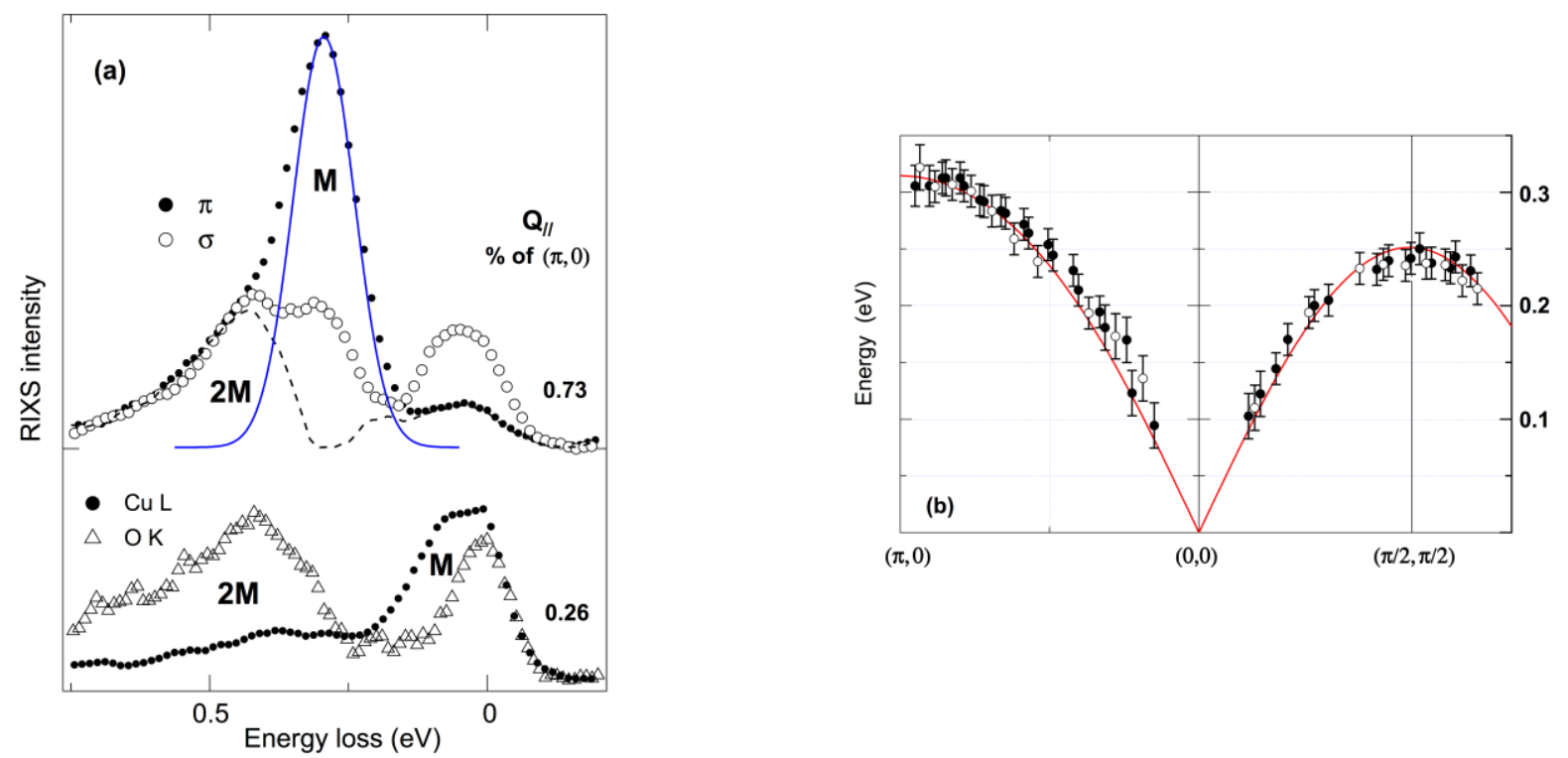

Figure 5. (a) Top: $\mathrm{Cu} \mathrm{L}_{3}$ RIXS of SCOC for two polarizations. Bottom: Comparison of $\mathrm{Cu} \mathrm{L}_{3}$ and $\mathrm{O} \mathrm{K}$ RIXS spectra for the same transferred momentum Q//. (b) Magnon dispersion, compared with the prediction of an extended Heisenberg model. From Ref. [16].

The capabilities of RIXS as a probe of magnetic excitations are well illustrated by data for the compound $\mathrm{Sr}_{2} \mathrm{CuO}_{2} \mathrm{Cl}_{2}$ (SCOC) [16]. SCOC is an $\mathrm{AFM}$ Mott insulator $\left(\mathrm{T}_{\mathrm{N}}=256 \mathrm{~K}\right)$ characterized by weakly interacting $\mathrm{CuO}_{2}$ layers. It is considered an almost ideal realization of an $\mathrm{S}=1 / 2$ square lattice Heisenberg AFM [17], with reduced $\left(\mu=0.34 \mu_{\mathrm{B}}\right)$ moments aligned along the (110) direction, interacting with an exchange energy $J=0.13 \mathrm{eV}$. Figure 5 (a) shows $\mathrm{Cu}_{3} \mathrm{~L}_{3}$ data measured close to $(\pi, 0)$ with two different linear polarizations. The spectra exhibit strong polarization dependence. The spectrum for $\pi$ polarization (in the scattering plane) is dominated by the single magnon peak $(\mathrm{M})$, which is fitted by a resolution-limited Gaussian line shape. The high-energy tail is due to the two-magnon continuum (2M; dashed line), while the weak elastic 
line is broadened by unresolved phonon sidebands. As expected from theory, the intensity of the magnon peak is strongly reduced in the spectrum measured with $\sigma$ polarization, which more clearly shows the two-magnon tail. $\mathrm{Cu} \mathrm{L}_{3}$ and $\mathrm{O} \mathrm{K}$ edge data are compared in the bottom part of the figure for $\mathrm{Q}_{/ /}=0.26(\pi, 0)$, closer to the $\mathrm{BZ}$ center, consistent to the smaller momentum window available at the oxygen edge. The signature of the magnon, now a distinct shoulder at $\sim 0.12 \mathrm{eV}$ in the $\mathrm{Cu}$ spectrum, is absent at the $\mathrm{O} \mathrm{K}$ edge spectrum where the spin-flip excitation is forbidden. The two-magnon contribution, on the other hand, is well visible, in agreement with with hard X-ray data from the $\mathrm{Cu} \mathrm{K}$-edge [18].

The full magnon dispersion in SCOC is shown along two high-symmetry directions of the BZ in Fig. 5(b). As expected, maxima are observed at the boundaries of the magnetic BZ. According to linear spin wave theory the value at $(\pi, 0)$, including quantum corrections, is E 2.36 $J$, which directly yields $J=130 \mathrm{meV}$. The large $(60 \mathrm{meV})$ energy difference between the $(\pi, 0)$ and $(\pi / 2, \pi / 2)$ points is remarkable, because the simple Heisenberg model with nearest-neighbour (NN) exchange, which is consistent with INS data around $(\pi, \pi)$ and is supposed to describe the physics of SCOC, does not predict any dispersion along the zone boundary. Quantum effects actually introduce a dispersion, but in the opposite direction [19]. This observation reveals a fundamental limitation of the NN Heisenberg model, as was already suggested by similar INS results for LCO [20]. The observed dispersion is reproduced when second- and third-neighbor $\left(J^{\prime}, J^{\prime \prime}\right)$ as well as 4-spin ring exchange $\left(J_{c}\right)$ interactions are considered, with $J=142 \mathrm{meV}, J^{\prime}=J^{\prime \prime}=4$ $\mathrm{meV}$, and $J_{c}=89 \mathrm{meV}$. The extended magnetic interactions can actually be derived from the more fundamental and purely electronic $t-t^{\prime}-t^{\prime \prime}-U$ extended Hubbard model. This result is interesting because it clearly shows the importance of extended electronic hopping terms in the electronic structure of the cuprates.

It should be stressed that complete dispersion curves such as in Fig. 5 (b) have been obtained by INS, the standard probe of spin waves, only recently and for a very limited number of cuprate materials. In this respect, the two techniques are really complementary. The advantages of INS are the broader momentum range, namely around the $(\pi, \pi)$ AFM wave vector, a better energy resolution, and an absolute determination of the cross section. RIXS, on the other hand, can easily probe large excitation energies, wave vectors close to $(0,0)$ where the neutron intensity is suppressed, and is more sensitive to the two-magnon continuum. Perhaps more important from a practical point of view, RIXS requires very small sample volumes, many order of magnitudes smaller than the typical single crystal sample for INS. This advantage is crucial for many complex or novel materials for which it may be impossible to obtain large single crystals of sufficient quality and homogeneity, and especially for phases that are not stable as bulk crystals and can only be synthesized as thin films. Dean et al. have exploited the large sensitivity of RIXS to investigate the spin response of a single cuprate layer in $\mathrm{La}_{2} \mathrm{CuO}_{4}-\mathrm{LaAlO}_{3}$ superlattices [21]. They found that the magnon excitations of the bulk crystal survive essentially unaffected in very thin LCO layers, down to the thickness of a single unit cell. This remarkable result shows that spin wave theory for the Heisenberg model provides a good description even for a single $\mathrm{CuO}_{2}$ layer, where one could expect quantum fluctuations to dominate the spin response. 
RIXS measurements at the $\mathrm{O} K$ edge do not give access to single spin-flip transitions, but can still provide useful information on the two-magnon states, complementary to that obtained at the L edge [22,23]. The two-magnon spectrum is actually a structured continuum, and RIXS probes it in different ways at the two edges, because matrix elements are different. Comparison of theory and experiment in LCO shows that O K-edge RIXS is mainly sensitive to the upper ridge of the continuum. The nearly Q-independent energy of the corresponding spectral feature would be $\sim 4 J$ in the non-interacting limit, but is reduced by the magnon-magnon interaction. By contrast, the two-magnon signal in $\mathrm{Cu}$ L-edge RIXS mainly reflects the strongly dispersive lower boundary of the same continuum. The two measurements are therefore complementary. At the $\mathrm{O}$ $\mathrm{K}$ edge, the absence of an overlapping single magnon signal makes it easier to study the continuum, but the price to pay is a smaller momentum window, less than half of the typical BZ of the cuprates. The $\mathrm{O} \mathrm{K}$ edge spectrum is also complementary to INS because the intensity of the two-magnon signal remains appreciable also around $(0,0)$, where the INS signal is strongly suppressed.

\section{- Superconducting cuprates}

The extension of RIXS measurements to doped SC cuprates in the past 5 years has produced interesting and somewhat unexpected results. They complement data from traditional magnetic probes (INS, NMR) and challenge our understanding of the electronic and magnetic properties of these fascinating materials. According to "magnetic scenarios" for high-Tc superconductivity, the spin excitations of the AFM insulating cuprate parent compounds are expected to survive as short-range fluctuations in the doped materials and lead to Cooper pair formation [24]. The predicted persistence of spin excitations in the superconducting part of the phase diagram was first confirmed by Braicovich et al. [25] in $\mathrm{Cu} \mathrm{L}_{3}$ measurements of underdoped LSCO. They found losses that tracked the magnon dispersion of AFM LCO, but also a lower-energy branch, suggesting the presence of a dynamic phase separation, possibly compatible with the formation of stripes. The similarities between parent AFM insulators and SC compounds have been further evidenced by Le Tacon et al. [26] who performed comprehensive RIXS measurements of magnetic excitations in various members, from underdoped to slightly overdoped, of the NdBCO and YBCO family. Dispersing excitations are observed in all compounds. Again, their energies closely follow the dispersion of the insulating parent compound, suggesting that the exchange energy $J$ is essentially independent of the doping level. Their line width, on the other hand, is substantially broader than in the insulator. These features were interpreted as representing paramagnon excitations, i.e. spin waves strongly damped by the interaction with the Stoner continuum of electron-hole excitations. Their spectral weight, integrated over energy and momentum, is essentially doping independent, and very close to the spectral weight of the magnon in the insulator. Spin excitations in SC cuprates had been previously observed and studied by INS, but only at much lower energy (40-60 meV), around the AFM wave vector $(\pi, \pi)$, where they give rise to the well-known hourglass anomaly. Their low integrated spectral weight, representing only a small fraction of the integrated magnon spectral weight of insulating cuprates such as LCO, has been difficult to reconcile with the idea of a magnetic "glue" for pairing. The RIXS data indicate that the missing spectral weight is recovered at larger energies and momenta. 
Solving the Eliashberg function for the measured spectrum in the framework of the $t$ - $J$ model yields a critical temperature $\mathrm{T}_{\mathrm{c}} \sim 200 \mathrm{~K}$ that is compatible, within a factor 2 , with the experimental values. This semi-quantitative agreement has been taken as a strong element in support of the magnetic scenario.

Similar damped paramagnon line shapes and dispersions have subsequently been measured in other cuprate families and over a broad range of doping levels, including highly overdoped Tl2201 samples [27]. Dean et al. [28] have measured LSCO thin film samples, where an accurate control of the doping level is possible over a broad doping range spanning the entire phase diagram, with atomically smooth surfaces that reduce the incoherent elastic scattering. Magnetic excitations are visible at all doping levels, with the same dispersion and integrated intensity, but broadened by a factor 3 to 4 with respect to insulating LCO. Since for highly overdoped samples phase separation into metallic and non-magnetic and insulating and magnetic regions can be excluded, these results indicate that the spin excitations are intrinsic. They imply that spin correlations are unexpectedly robust, and extend into a region of the phase diagram where thermodynamic, transport and magnetotransport data are well described by a Fermi liquid scenario, and $\mathrm{T}_{\mathrm{c}}$ is considerably reduced. This is not necessarily in contradiction with a magnetic scenario, because the high-energy spin excitations are expected to contribute only weakly to Cooper pairing, unlike the low-energy spin excitations that build up near optimum doping around $(\pi, \pi)$. The comparison with INS is interesting. It shows that around the AFM wave vector probed by neutrons, spin excitations are suppressed by doping and the dispersion is strongly renormalized to form the "hourglass" feature, while - apart from the broadening - they are relatively unaffected at wave vectors near the center of the first BZ sampled by RIXS. This asymmetry is actually captured by calculations performed in the framework of the single-band Hubbard model. The data are consistent with INS and O K-edge RIXS data [29] that also reveal persistent high-energy magnetic excitations in overdoped LSCO.

Bismuth-based cuprates have been less extensively studied by INS than compounds of the YBCO and LSCO families, owing to the difficulty of obtaining large single crystals of sufficient quality. Knowledge of magnetic excitations is correspondingly less advanced, since INS studies have mainly be limited to the low-energy excitations around the AFM wave vector. On the other hand ARPES has exploited the superior quality of their cleaved surfaces, and revealed many fine details of the electronic structure [30]. This is especially true for bilayer Bi-2212, which therefore plays a prominent role among the cuprates. The much less demanding requirements on sample size has opened the way to RIXS investigations of the high-energy magnetic response in these materials. Dean et al. [31] have measured underdoped and optimally doped Bi-2212 (Fig. 6) and found that they also exhibit "paramagnon" line shapes similar to Ref. [26]. For the first time they used the incident polarization dependence of RIXS to demonstrate the spin-flip character of the MIR features, verifying that the intensity is enhanced at grazing emission with $\pi$ polarization, as predicted by theory. A good fit of the magnon dispersion is obtained with the spin susceptibility calculated with the phenomenological model of the electronic structure of Yang, Rice and Zhang, using a parameter set that describes the quasiparticle bands known with high accuracy from ARPES. Furthermore, the largest energy of the spin excitations $(\sim 340 \mathrm{meV}$, 
at the zone boundary) is precisely the energy of the anomaly (the "kink") of the quasiparticle band revealed by ARPES. This close connection suggests that it should be possible to obtain a unified description of electronic and magnetic properties of cuprates. Dispersive paramagnon line shapes were also observed for the other members of the Bi-based family, but only along the antinodal $(0,0) \rightarrow(\pi, 0)$ direction. Here, the zone-boundary energy increases along with $T_{c}$ from $300 \mathrm{meV}$ in single-layer $\mathrm{Bi}-2201$ to $350 \mathrm{meV}$ in triple-layer $\mathrm{Bi}-2223$, which indicates an increasing exchange interaction. By contrast, the spectra in the nodal $(0,0) \rightarrow(\pi, \pi)$ direction exhibit diffuse and weakly dispersive excitations that do not seem to be consistent with the paramagnon scenario [32]. The asymmetry of the data along the two directions is at least qualitatively reproduced by the magnetic susceptibility calculated in a renormalized itinerant quasiparticle approach. This provides an indication that the nature of magnetism in the cuprates is probably at least partly itinerant.

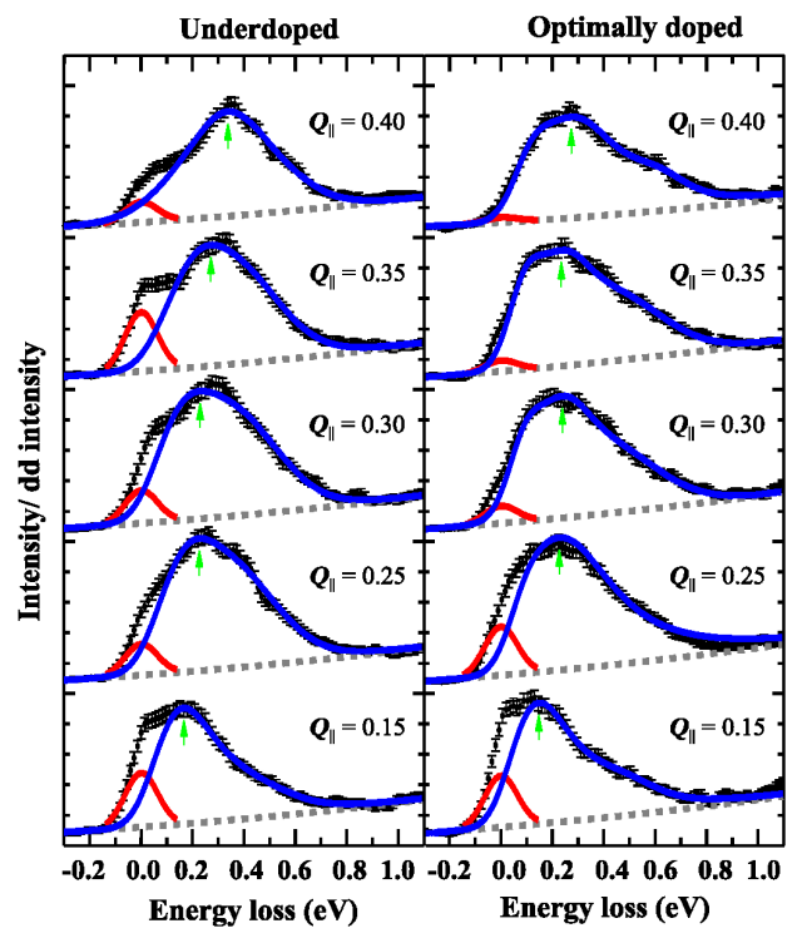

Figure 6. $\mathrm{Cu} \mathrm{L}_{3}$ RIXS spectra of underdoped (left) and optimally doped (right) Bi-2212, from [31].

A completely itinerant scenario has been advocated by Benjamin et al. [33], who challenge the generally accepted assignment of the dispersing features in RIXS of SC cuprates to collective magnetic modes. They used an original RIXS formalism for non-interacting particles, and the band structure of a typical cuprate material, to calculate the RIXS spectral function. The calculation shows that band structure alone can produce broad spectral features with comparable intensities in both the spin flip and the non-spin flip channels. This approach naturally yields spectra that are quite insensitive to the doping level, as required by the experiments described above, since a small change of the chemical potential has little effect on the band structure. It also predicts that the low-energy features should exhibit non-Raman behaviour above threshold, i.e. they should disperse and exhibit an evolution with incident energy. Guarise et al. [34] took a less extreme point of view in the interpretation of results from underdoped and optimally doped 
Bi-2212 samples. In the antinodal $(0,0)$ to $(\pi, 0)$ direction the data show dispersive features similar to the magnons of the parent compound, in agreement with other published data. By contrast, along the nodal $(0,0)$ to $(\pi, \pi)$ direction these excitations soften considerably and extend down to zero energy loss. Such anisotropy, which was largely ignored in previous analyses, suggests that the scenario of damped paramagnons, built on AFM-correlated local spins, may not completely describe the SC materials. Above threshold the data exhibit distinctive non-Raman behaviour, indicative of a continuum of excited states, rather than collective excitations. Such non-Raman dispersion is not unique to $\mathrm{Bi}-2212$, and has later been observed also in LSCO, as shown in Fig. 7 [35]. The main features of the experimental results can be reproduced by the RPA spin susceptibility for a single band Hubbard model, i.e. the spin susceptibility of a noninteracting metal, renormalized by the Coulomb interaction. This underpins a scenario of collective spin excitations built from correlated itinerant electrons rather than from local spins. Interestingly, the RPA approach yields similar spectral line shapes in the spin flip and non-spin flip (charge) channels as in [33], since they both arise from the same electron-hole excitations across the Fermi surface. However, unlike [33], the spin channel is enhanced while the charge channel is suppressed by the RPA renormalization, and therefore they are expected to exhibit quite different intensities, namely the spin channel should be dominant.

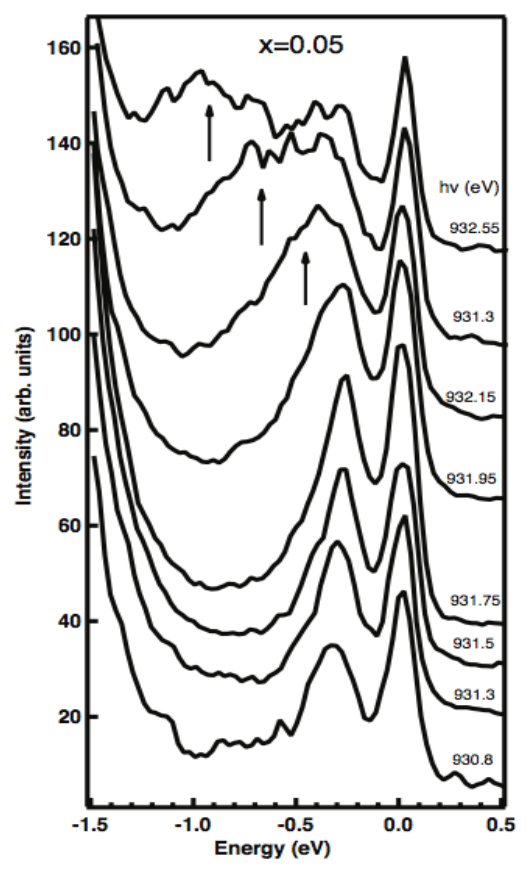

Figure 7. $\mathrm{Cu} \mathrm{L}_{3}$ RIXS spectra of a strongly underdoped ( $\mathrm{x}=0.05$ ) LSCO sample measured near $(\pi, 0)$ for incident energies spanning the resonance. Arrows point to a non-Raman feature [35].

The persistence of high-energy spin excitations in $\mathrm{Cu} \mathrm{L}_{3}$ RIXS spectra well into the overdoped part of the phase diagram, where superconductivity is suppressed, contrasts with INS and Raman results, and seems to challenge the magnetic scenario of superconductivity. This apparent inconsistency has been examined by Jia et al. [36] based on a numerical treatment of an effective one-band extended $t-t^{\prime}-U$ Hubbard model. The results of an exact diagonalization first of all 
show that the RIXS spectral function of doped cuprates, even without polarization analysis of the scattered beam, is always quite close to the spin dynamical structure factor $S(\mathbf{q}, \omega)$, and is therefore directly comparable with other probes of magnetism, namely INS. The coherent spin waves are rapidly suppressed by doping, which destroys the AFM order, and $S(\mathbf{q}, \omega)$ is rapidly modified in the region of momentum space near $(\pi, \pi)$ probed by INS, where a spin gap opens. By contrast, short-range spin correlations survive to high doping levels. Spin excitations, at the energy scale of $J$, are therefore relatively unaffected, but probably do not play a major role in the pairing mechanism. Interestingly, at the magnetic zone-boundary the energy of the paramagnon slightly softens upon hole doping, but it is seen to harden by as much as $50 \%$ for electron doping.

The asymmetry between electron and hole doping suggested by the Hubbard model calculation has been verified in experiments by two different groups. Ishi et al. [37] have measured the electron-doped compounds $\mathrm{Nd}_{2-\mathrm{x}} \mathrm{Ce}_{\mathrm{x}} \mathrm{CuO}_{4}(\mathrm{NCCO})$ and (Pr-La) $2-\mathrm{x} \mathrm{Ce}_{\mathrm{x}} \mathrm{CuO}_{4}(\mathrm{PLCCO})$, at various doping levels from an AFM insulator to an overdoped superconductor. The comparison of $\mathrm{L}_{3}$ RIXS with two different polarizations, K-edge RIXS and INS results enables a separation of magnetic excitations, with energies of order $J$, and charge excitations at higher energy of order $t \sim 400 \mathrm{meV}$. Unlike the case of hole-doped cuprates, the spin-wave excitations of the insulator indeed harden with doping. This is taken as an indication of the more itinerant nature of electrondoped cuprates. The charge and spin excitations merge in the optimally-doped and overdoped samples. This suggests that the paramagnon concept is less well-grounded here, and that the spin flip excitations can be considered to form a sort of Stoner continuum. Lee et al. [38] also find in NCCO a hardening up to $50 \%$ of the magnetic mode relative to the insulator, with an energy of up to $450 \mathrm{meV}$ at the AFM zone boundary. They also observe a new mode rapidly dispersing from the zone center with a velocity similar to the Fermi velocity, which is a strong indication of a charge origin. This mode persists well above $T_{c}$ in the SC samples. It provides evidence of a different ordered state, such as a charge- or spin-density-wave. At the same time, the intensity of the spin flip excitations in the e-doped SC samples does not follow the reduction of the SC $\mathrm{T}_{\mathrm{c}}$, at variance with the case of hole-doped materials.

To summarize this section, soft X-ray RIXS results on doped cuprates have considerably enlarged the phenomenology of the spin dynamics in these materials. The spectra show both itinerant and local aspects, as e.g. in the data of Fig. 7. At this time, despite the success of the paramagnon scenario, a clear consensus on their interpretation has not yet been achieved. A very recent experiment [39] on YBCO stresses the importance of the polarization and scattering geometry to selectively enhance spin or charge contributions. Exploiting these parameters in a systematic way in various cuprate families and for a broad range of doping levels should clarify this crucial issue. This work is already in progress. From the point of view of theory, some version of the Hubbard model probably contains the relevant physics of the SC cuprates. Nevertheless its solution in the most general case, including the spectral properties, remains out of reach. The "local" and "itinerant" scenarios represent two manageable limits of the full theory. The challenge ahead is how to bridge the gap between these two limits. In this perspective, future extensive RIXS (and INS) data will certainly provide a precious guideline. 


\section{- Other materials}

Besides the high- $\mathrm{T}_{\mathrm{c}}$ cuprates, and their insulating parent compounds, soft X-ray RIXS experiments in the past five years have addressed the spin dynamics in other classes of materials, such as quasi one-dimensional (1D) oxides, the iron pnictide superconductors and the iridates.

\section{$1 D$ oxides}

The hallmark of the electronic structure of interacting fermions in one dimension is the instability of the Landau Fermi liquid [40]. Quasiparticles in 1D are not stable: they split into collective excitations carrying either charge (holons) or spin (spinons) and propagating at different velocities. Ground breaking soft X-ray RIXS measurements of the "telephone number" compound $\mathrm{Sr}_{14} \mathrm{Cu}_{24} \mathrm{O}_{41}$ [41] and of the quantum magnet $\mathrm{TiOCl}$ [42] have indeed found the typical 1D signatures of spin excitations, independently characterized by INS. A more recent $\mathrm{Cu}$ L-edge RIXS experiment on the $1 \mathrm{D}$ oxide $\mathrm{Sr}_{2} \mathrm{CuO}_{3}$ [43] has revealed a different kind of instability, involving the orbital degrees of freedom. The RIXS spectra show dd excitations at 2$3 \mathrm{eV}$ energy loss, and low-energy spin excitations, as in 2D cuprates, but the momentum dependence of the two kinds of excitations is distinctively different. The spin excitations define a continuum, dispersing with different momentum periodicities for the lower $(\pi / a)$ and upper ( $2 \pi / a)$ boundaries. These are the characteristic signatures of the spinon, consistent with INS data, including the extracted large exchange coupling $J=249 \mathrm{meV}$. Unlike the $2 \mathrm{D}$ cuprate case, the dd manifold exhibit orbital-selective dispersions and intensity modulations. They are well captured by a $1 \mathrm{D} J_{O}-J$ model, the analogue of the $t-J$ model where the charge hopping term $t$ is replaced by an orbital exchange $J_{O}$. It describes an underlying physical picture where the RIXS process creates a spin excitation together with a dd excitation, and the latter can separate itself from the spinon and propagate through the lattice as an "orbiton", i.e. a collective orbital excitation.

Bisogni et al. [44] have exploited $\mathrm{Cu}$ L-edge RIXS to probe the femtosecond dynamics of magnetic excitations in the $1 \mathrm{D}$ two-leg ladder compound $\mathrm{CaCu}_{2} \mathrm{O}_{3}$. The momentum- and polarization dependence of the RIXS line shape, supported by a theoretical calculation of the cross section, allow the single- and double spin flip $(\Delta S=0)$ excitations to be disentangled, despite their partial overlap, and the much smaller (10 to 20 times) intensity of the latter. From the relative intensities it is possible to estimate the characteristic times over which the corresponding excitations are created. Here the "timing" is set by the core-hole lifetime $\tau$ (the socalled core-hole clock). An exact diagonalization on the Heisenberg model for a spin-1/2 AFM chain that explicitly incorporates the core-hole lifetime, indicates that the single spin-flip excitations are created upon decay of the core-hole state, and are quite insensitive to the exchange energy $J$ or $\tau$. The picture is different for the double spin flips, which are created because the core-hole site introduces a spin vacancy in the AFM chain. The characteristic time for this process is $\tau_{S_{0}}=\hbar / J$ and depends on the intermediate state dynamics. Therefore, the double spin flip intensity is appreciable only when $\tau$ is comparable with $\hbar / J$ and increases with $\tau$. A fit to the data yields $s_{0} 4 \mathrm{fs}(J=0.16 \mathrm{eV})$ for $\mathrm{CaCu}_{2} \mathrm{O}_{3}$, and 2 fs. These results show that RIXS gives access to the ultrafast spin dynamics in 1D materials. 
Even if they cannot directly measure single spin excitations, RIXS data at the oxygen K edge can indirectly probe magnetic correlations with exquisite sensitivity. This is demonstrated by measurements of the $1 \mathrm{D}$ edge-sharing cuprate systems $\mathrm{Li}_{2} \mathrm{CuO}_{2}$ and $\mathrm{CuGeO}_{3}$ [45]. The spectra of both compounds exhibit a feature at $\sim 3.5 \mathrm{eV}$ energy loss, intermediate between the dd and charge-transfer excitations, corresponding to a Zhang-Rice singlet (ZRS) final state. The ZRS, well characterized by ARPES in 2D cuprates, has two holes with opposite spins on a $\mathrm{CuO}_{4}$ plaquette, and one filled $3 \mathrm{~d}$ shell in the neighboring plaquette, or in short a $\mathrm{d}^{9} \underline{\mathrm{L}} ; \mathrm{d}^{10}$ configuration. A theoretical analysis shows that the intensity of the ZRS in RIXS depends in a crucial way on the relative arrangements of the $\mathrm{Cu}$ spins. Namely, it is suppressed if the spins are parallel, as in the FM ground state of $\mathrm{Li}_{2} \mathrm{CuO}_{2}$, and allowed for an antiparallel spin arrangement, as in the ground state of the spin-Peierls system $\mathrm{CuGeO}_{3}$. In both cases quantum fluctuations at $\mathrm{T}=0$, and thermal fluctuations at finite temperature, populate excited states with different spin arrangements. As a consequence, the ZRS has nonzero intensity even at the lowest measurement temperature of $9 \mathrm{~K}$. However its temperature evolution is distinctly different for the two materials. The intensity increases with increasing temperature in $\mathrm{Li}_{2} \mathrm{CuO}_{2}$, but decreases with increasing temperature in $\mathrm{CuGeO}_{3}$, showing that RIXS can probe magnetic correlations at the $\mathrm{meV}$ scale, irrespective of an experimental resolution that is almost two orders of magnitude larger.
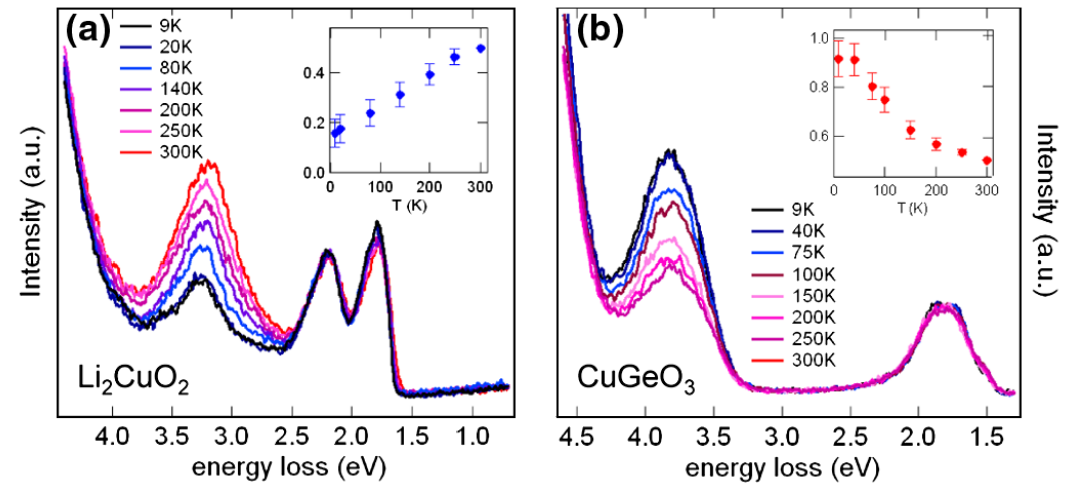

Figure 8. O K RIXS spectra show that the intensity of the ZRS feature increases with temperature for $\mathrm{FM} \mathrm{Li}_{2} \mathrm{CuO}_{2}$ (left) and decreases for $\mathrm{AFM} \mathrm{CuGeO}_{3}$ [45].

\section{Pnictides}

The Fe-based pnictide and chalcogenide superconductors share with the SC cuprates an intriguing proximity to a magnetic phase. However, the parent compounds are semi-metals rather than (charge-transfer) insulators. Different scenarios have been proposed to describe the nature of the magnetic phases. A weak-coupling scenario invokes nesting of electron and hole Fermi surfaces as the origin of a magnetic instability. The alternative strong-coupling scenario is based on large fluctuating local spins. Fe L-edge RIXS measurements must cope with a smaller momentum window than at the $\mathrm{Cu}$ edge, but can take advantage of a better energy resolution. Data from the AFM parent compound $\mathrm{BaFe}_{2} \mathrm{As}_{2}$ (BFA) [46] reveal, similarly to the cuprates, magnon features dispersing to up $200 \mathrm{meV}$ at the magnetic BZ boundary, in good agreement 
with INS data. Unlike the cuprate parent compounds, however, the line widths are not resolution limited, which probably reflects damping due to the interaction with itinerant electrons. RIXS data for optimally hole-doped $\mathrm{SC} \mathrm{Ba}_{0.6} \mathrm{~K}_{0.4} \mathrm{Fe}_{2} \mathrm{As}_{2}$ (BKFA; $\mathrm{T}_{\mathrm{c}}=39 \mathrm{~K}$ ) show similarly well-defined "paramagnon" excitations, albeit slightly softened, with a zone-boundary energy of $150 \mathrm{meV}$, and similar spectral weight. Moreover, the spectra are very similar in the SC and in the normal state. These observations support the strong-coupling scenario of a correlated spin state with short-range spin correlations over the Fermi-surface nesting picture.

\section{Iridates}

The perovskite iridate materials are oxides containing $\operatorname{Ir}^{4+}$ ions with a $\mathrm{d}^{5}$ local electronic configuration. The spin-orbit interaction (SOI), which is considerably larger than for the $3 d$ electrons of the cuprates, splits the Ir $5 \mathrm{~d} \mathrm{t}_{2 \mathrm{~g}}$ manifold into an occupied fourfold degenerate and a half-filled doubly degenerate state. The latter can be described by a $J=1 / 2$ isospin, and exhibits a strong analogy with the $S=1 / 2$ state of the cuprates [47]. The excitations of this $J=1 / 2$ state have been studied by RIXS at the Ir $\mathrm{L}_{3}$ edge $(11.2 \mathrm{keV})$ in the hard X-ray region [48]. These results exhibit both clear analogies and interesting differences with the $\mathrm{Cu} \mathrm{L}_{3}$ RIXS data of the cuprates. An interesting recent theoretical development concerning the O K-edge [49] falls more directly into the scope of the present review. As already discussed, the mechanism that allows single spin-flip processes at an $\mathrm{L}$ edge is not active at $\mathrm{K}$ edges, because of the lack of SOI for the $1=0$ core hole. Nevertheless, single spin flip can still be observed at the $\mathrm{O} K$ edge of a transition metal (TM) oxide by a different mechanism, which depends on the TM-O hybridization. It requires i) SOI in the TM d-shell, and ii) broken inversion symmetry at the $\mathrm{O}$ site. Both conditions can be satisfied in the iridates, e.g. in the single-layer $\mathrm{Sr}_{2} \mathrm{IrO}_{4}$ and in the bi-layer $\mathrm{Sr}_{3} \mathrm{Ir}_{2} \mathrm{O}_{7}$, or in the post-perovskite $\mathrm{CaIrO}_{3}$, where inversion symmetry is broken due to a large buckling of the $\mathrm{IrO}_{6}$ octahedra. Soft X-ray RIXS is therefore expected to play a role also in this interesting class of materials. Strictly speaking, the same mechanism is also present in the cuprates, but the calculations show that its contribution to single spin-flip scattering is rapidly reduced with reduced SOI, to a point that it is essentially negligible in the cuprates.

\section{- Conclusions and perspectives}

Over the past 5 years RIXS has evolved from a somewhat exotic experiment into a routine probe of magnetism, complementary to well established techniques such as INS. Many interesting experimental results have been accumulated, especially in cuprate systems, and the field appears healthy and growing, as shown by many high-profile publications. There is certainly no sign of slowing down of the pace at which new physical insight is obtained, the main limitation being at present the limited amount of available beamtime. This bottleneck will be progressively removed as several planned RIXS beamlines, which will equal or surpass the performances of the SAXES instrument, are built at all major SR laboratories worldwide. The resulting increase of available beamtime will enable broader and more systematic investigations of a wide range of interesting materials. 
This remarkable progress has been boosted by the advent of high resolution, namely at the SAXES instrument. There is still room for future experimental improvements that take advantage of cutting-edge progress in X-ray source quality and optimization, optics technology and detectors. Undoubtedly, progress in high resolution will again bring along qualitative advances. The frontier will soon move from the present $130 \mathrm{meV}$ at the $\mathrm{Cu} \mathrm{L}$-edge to $20-30 \mathrm{meV}$ or better, with the commissioning of new RIXS instruments at several light sources, e.g. at the ESRF [50], BESSY-II [51], DIAMOND [52], MAX-IV [53] and NSLS-II [54] and NSRRC [55]. At that level it will be possible to disentangle magnons from phonons, and to follow their dispersion still closer to $\mathrm{Q}=0$, for a more accurate analysis. Another important step for the study of magnetic materials will be the possibility of routinely performing a polarization analysis of the outgoing beam [50] with good resolution and intensity. Such capability has long been a crucial asset in elastic magnetic X-ray scattering. As discussed in a previous section, both theoretical considerations and experimental evidence indicate that it will also play a major role in future RIXS experiments. Finally, RIXS is bound follow the pathway of other techniques (ARPES, optics, diffraction) towards ultrafast pump-probe experiments. Such challenging experiments are just becoming possible thanks to the advent of powerful free-electron-laser sources. They will directly address excitations in the time domain, and also enable the study of transient phases and, if successful experimental schemes can be devised, open the way to stimulated RIXS experiments, with possible gains in intensity and time-resolution tunability that still need to be explored.

\section{Acknowledgements}

It is a pleasure to acknowledge collaborations and illuminating discussions with L. Braicovich, B. Dalla Piazza, I. Eremin, G. Ghiringhelli, M. Guarise, H. Rønnow, G.A. Sawatzky, T. Schmitt, J. van den Brink, and the financial support of the Swiss NSF. 


\section{References}

1. W. Shülke, Electron Dynamics by Inelastic X-Ray Scattering (Oxford University Press, Oxford, 2007).

2. A. Kotani and S. Shin, Rev. Mod. Phys. 73, 203 (2001).

3. L.J.P. Ament, M. van Veenendaal, T.P. Devereaux, J.P. Hill, J. van den Brink, Rev. Mod. Phys. 83, 705 (2011).

4. M. van Veenendaal, Theory of Inelastic Scattering and Absorption of X-rays, (Cambridge University Press, 2015).

5. Soft X-ray Emission Spectroscopy, E.J. Nordgren and E.Z. Kurmaev eds., J. El. Spectr. Rel. Phenom. 110-111 (2000).

6. K. Ishii, T. Tohyama, and J. Mizuki, J. Phys. Soc. Jpn. 82, 021015 (2013).

7. G. Ghiringhelli et al., Rev. Sci. Instrum. 77, 113108 (2006).

8. F. M. F. de Groot, P. Kuiper, and G. A. Sawatzky, Phys. Rev. B 57, 14584 (1998).

9. P. Kuiper et al., Phys. Rev. Lett. 80, 5204 (1998).

10. S. G. Chiuzbaian et al., Phys. Rev. Lett. 95, 197402 (2005).

11. G. Ghiringhelli et al., Phys. Rev. Lett. 102, 027401 (2009).

12. L. Braicovich et al., Phys. Rev. Lett. 102, 167401 (2009).

13. L.J.P. Ament et al., Phys. Rev. Lett. 103, 117003 (2009).

14. M.W. Haverkort et al., Phys. Rev. Lett. 105, 167404 (2010).

15. J.-I. Igarashi and T. Nagao, Phys. Rev. B 85, 064421 (2012).

16. M. Guarise et al., Phys. Rev. Lett. 105, 157006 (2010).

17. Greven, Z. Phys. B 96, 465 (1995).

18. J.P. Hill et al., Phys. Rev. Lett. 100, 097001 (2008).

19. N.B. Christensen et al., Proc. Natl. Acad. Sci. U.S.A. 104, 15264 (2007).

20. R. Coldea et al., Phys. Rev. Lett. 86, 5377 (2001).

21. M.P.M. Dean et al., Nat. Materials 11, 850 (2012).

22. Y. Harada et al., Phys. Rev. B 66, 165104 (2002).

23. V. Bisogni et al., Phys. Rev. B 85, 214527 (2012).

24. D.J. Scalapino, Rev. Mod. Phys. 84, 1383 (2012).

25. L. Braicovich et al., Phys. Rev. B 104, 077002 (2010).

26. M. Le Tacon et al., Nat. Phys. 7, 725 (2011).

27. M. Le Tacon, Phys. Rev. B 88, 020501(R) (2013).

28. M.P.M. Dean et al., Nat. Materials 12, 1019 (2013).

29. V. Bisogni et al., Phys. Rev. B 85, 214528 (2012).

30. A. Damascelli, Z. Hussain, and Z.-H. Shen, Rev. Mod. Phys. 75, 473 (2003). 
31. M.P.M Dean et al., Phys. Rev. Lett. 110, 147001 (2013).

32. M.P.M. Dean et al., Phys. Rev. B 90, 220506(R) (2014).

33. D. Benjamin et al., Phys. Rev. Lett. 112, 247002 (2014).

34. M. Guarise et al., Nat. Commun. 5, 5760 (2014).

35. S. Fatale et al., in preparation.

36. C.J. Jia et al., Nat. Commun. 5, 3314 (2014).

37. K. Ishi et al., Nat. Commun. 5, 3714 (2014).

38. W.S. Lee et al., Nat. Phys. 10, 883 (2014).

39. M. Minola et al., arXiv:1502.02583v1.

40. T. Giamarchi, Quantum Physics in One Dimension (Clarendon Press, 2004).

41. J. Schlappa et al., Phys. Rev. Lett. 103, 047401 (2009).

42. S. Glawon et al., Phys. Rev. Lett. 107, 107402 (2011).

43. J. Schlappa et al., Nature 485, 82 (2012).

44. V. Bisogni et al., Phys. Rev. Lett. 112, 147401 (2014).

45. C. Monney et al., Phys. Rev. Lett. 110, 087403 (2013).

46. K.J. Zhou et al., Nat. Commun. 4, 1470 (2013).

47. B.J. Kim et al., Phys. Rev. Lett. 101, 076402 (2008).

48. J. Kim et al., Phys. Rev. Lett. 108, 177003 (2012); ibid. 109, 157402 (2012).

49. B.H. Kim and J. van den Brink, arXiv:1404.2040v1.

50. http://www.esrf.eu/UsersAndScience/Experiments/ElectStructMagn/ID32.

51. https://www.helmholtzberlin.de/pubbin/igama_output?modus=einzel\&sprache=en\&gid=1868\&typoid=40737.

52. http://www.diamond.ac.uk/Beamlines/Spectroscopy/I21.html.

53. https://www.maxlab.lu.se/veritas.

54. http://www.bnl.gov/ps/nsls2/beamlines/files/pdf/02ID-SIX.pdf

55. C.H. Lai et al., J. Synchrotron Rad. 21, 325 (2014). 